\title{
A Nonlinear Application of a Lyapunov Technique for Assuring Global Performance Bounds
}

\author{
Anders Helmersson \\ Department of Electrical Engineering \\ Linköping University \\ S-581 83 Linköping, Sweden \\ email: andersh@isy.liu.se \\ fax: +4613282622
}

\begin{abstract}
In this paper we consider the stability and performance problem of nonlinear systems using a Lyapunov technique. Upper or lower bounds can be provided assuming that a Lyapunov function satisfies a Hamiltonian inequality for all admissible states. Also, suboptimal control laws with guaranteed performance bounds can be derived with this technique. The technique has been applied on rate and attitude control for sounding rockets. As an example a rate control algorithm is elaborated showing the main ideas presented in this paper: 1) the Lyapunov function provides a global performance bound; 2) a control law is derived based on the Lyapunov function; and 3) the candidate Lyapunov function is parametrized and improved bounds are obtained using parameter optimization.
\end{abstract}

Keywords: Optimal control, suboptimal control, performance bounds, Lyapunov functions, nonlinear systems.

\section{Introduction}

Many control problems can be formulated as optimization problems using a performance or cost criterion. In practical applications it is usually very difficult or even impossible to synthesize this into a tractable control law. Instead of finding the optimal control law it is normally easier to find a suboptimal strategy. This can be facilitated by turning the Hamilton-Jacobi-Bellman equation [1] into an inequality. In this paper we will apply a Lyapunov technique for providing bounds of performance for nonlinear systems. A similar methodology is used for dissipative systems where the storage function serves as a Lyapunov function (see eg. [2], [3] and [4]).
The technique is applied on a rate control system for a spacecraft. Similar control systems have been designed, implemented and flown on sounding rocket applications.

The paper is outlined as follows: Section 2 provides tools for proving upper and lower bounds of integral performance criteria. An example is given in section 3 showing the application of the technique on a rate control system for a spacecraft.

\section{Performance Bounds}

Consider a continuous-time dynamic system described by a differential equation

$$
\dot{x}=f(x, u)
$$

and a performance criterion $J$ defined by

$$
J(x(t))=\int_{t}^{T} g(x, u) d \tau
$$

where $x$ is the state vector and $u$ is the control input.

The performance criterion can be defined either for a given feedback control law, $u$, or as a minimization criterion. In the latter case a control law, minimizing the performance criterion is defined. In both cases it is generally not possible to find an explicit solution of $J$. Instead it may be possible to find a bound, $V$, that defines an upper limit of solution, that is $J \leq V$.

Theorem 2.1 An upper bound for $J$ can be established if there exists a continuously differentiable, positive definite Lyapunov function, $V$, and a control law, $u(x)$, that make the Hamiltonian, $H$, nonpositive for all $x$.

$$
H=g(x, u(x))+V_{x}(x) f(x, u(x)) \leq 0, \forall x
$$


Proof.

$$
\begin{aligned}
J(x(t))= & \int_{t}^{T} g(x, u) d \tau \\
= & \int_{t}^{T} g(x, u) d \tau+V(x(T))-V(x(t)) \\
& +V(x(t))-V(x(T)) \\
\leq & \int_{t}^{T}[g(x, u)+\dot{V}(x)] d \tau+V(x(t)) \\
= & \int_{t}^{T}\left[g(x, u)+V_{x}(x) f(x, u)\right] d \tau+V(x(t)) \\
\leq & V(x(t)) .
\end{aligned}
$$

Remark 2.1 A lower bound can be established analogously, by assuming $H$ to be non-negative.

Remark 2.2 If a Lyapunov function establishes both an upper and a lower bound then it is optimal and satisfies $H=0$ globally. This is usually referred to as the Hamilton-Jacobi-Bellman equation, see e.g. [1].

Remark 2.3 A control law can be derived from $V$ by $u(x)=\arg \min _{u} H$. This means that if an upper bound has been established for a given control law, a new control law can be defined, possibly with improved performance.

Remark 2.4 If we have a set of Lyapunov functions, $V_{\theta}$ parametrized by $\theta$, such that every member in the set satisfies $H \leq 0$, then a new Lyapunov function, $V_{*}$, can be defined by

$$
V_{*}(x)=\min _{\theta} V_{\theta}(x) .
$$

\section{An Example}

\subsection{Approach}

In this example we start by assuming a Lyapunov function with unknown parameters. The parameters are chosen in order to fulfill the Hamiltonian inequality and a control law is derived by minimizing the Hamiltonian with respect to the control signal. We actually derive a set of Lyapunov functions and by finding the minimum of these a new Lyapunov function is derived, which is globally equal to or better than any of the Lyapunov functions within the original set.

\subsection{Problem Definition}

In this example we will consider a rate control system for a cylindrical spacecraft equipped with a number of control thrusters for attitude and rate control. The dynamics of a rigid body can be expressed as

$$
\begin{aligned}
I_{x} \dot{\omega}_{x} & =\left(I_{y}-I_{z}\right) \omega_{y} \omega_{z}+M_{x} \\
I_{y} \dot{\omega}_{y} & =\left(I_{z}-I_{x}\right) \omega_{z} \omega_{x}+M_{y} \\
I_{z} \dot{\omega}_{z} & =\left(I_{x}-I_{y}\right) \omega_{x} \omega_{y}+M_{z}
\end{aligned}
$$

where $I$ is the moment of inertia of the body, $\omega$ is its angular rates, and $M$ is the control torque. The coordinate system $(x, y, z)$ is assumed to coincide with the principal axes of inertia of the body.

The cylindrical body is assumed to be symmetric about the x-axis, that is $I_{y}=I_{z}=I_{\perp}$. Define $\eta=$ $I_{x} / I_{\perp}, a_{x}=M_{x} / I_{x}, a_{y}=M_{y} / I_{\perp}$ and $a_{z}=M_{z} / I_{\perp}$. We then get the following set of simplified equations

$$
\begin{aligned}
& \dot{\omega}_{x}=a_{x} \\
& \dot{\omega}_{y}=(1-\eta) \omega_{z} \omega_{x}+a_{y} \\
& \dot{\omega}_{z}=(\eta-1) \omega_{x} \omega_{y}+a_{z} .
\end{aligned}
$$

As an application we choose a spacecraft configuration with three thruster pairs placed with an equal 120-degree spacing around the circumference of the spacecraft. The thrusters in each thruster pair produce opposite torques. The six available commands are

$$
\bar{a}=\left(a_{x}, a_{y}, a_{z}\right)=\left\{\begin{array}{c} 
\pm\left(\alpha_{x}, \alpha_{\perp}, 0\right) \\
\pm\left(\alpha_{x},-\frac{1}{2} \alpha_{\perp}, \frac{\sqrt{3}}{2} \alpha_{\perp}\right) \\
\pm\left(\alpha_{x},-\frac{1}{2} \alpha_{\perp},-\frac{\sqrt{3}}{2} \alpha_{\perp}\right)
\end{array}\right.
$$

where $\alpha_{x}$ and $\alpha_{\perp}$ are normalizing constants corresponding to the torque levels. These depend on the moments of inertia $\left(I_{x}\right.$ and $\left.I_{\perp}\right)$, the thrust level and the lever arm.

We use a cost function that penalizes both time and fuel consumption (accumulated thruster activation)

$$
g(x, u)=1+\lambda \sum_{i=1}^{6} u_{i},
$$

where $u_{i}$ are the control signals (either 0 or 1 ) and $\lambda \geq 0$ is the time-fuel tradeoff parameter (low $\lambda$ puts the emphasis on time and vice versa), which is used as a design parameter.

\subsection{Finding a Set of Lyapunov Function}

We adopt the following candidate Lyapunov function

$$
V(\bar{\omega})=\sqrt{b_{x}^{2} \omega_{x}^{2}+b_{\perp}^{2}\left(\omega_{y}^{2}+\omega_{z}^{2}\right)}
$$

where $b_{x}$ and $b_{\perp}$ are parameters to be determined. We normalize the equations by introducing $\bar{\Omega}=\left(\omega_{x} / \alpha_{x}, \omega_{y} / \alpha_{\perp}, \omega_{z} / \alpha_{\perp}\right)$, and $\bar{\beta}=$ 
$\left(b_{x} \alpha_{x}, b_{\perp} \alpha_{\perp}, b_{\perp} \alpha_{\perp}\right)$. The Lyapunov function can then be rewritten as

$$
V(\bar{\Omega})=\sqrt{\left(\beta_{x} \Omega_{x}\right)^{2}+\left(\beta_{\perp} \Omega_{\perp}\right)^{2}}
$$

where

$$
\Omega_{\perp}=\sqrt{\Omega_{y}^{2}+\Omega_{z}^{2}} .
$$

The Hamiltonian of the problem becomes

$$
H=1+\lambda \sum_{i=1}^{6} u_{i}+\frac{1}{V}\left(b_{x}^{2} \omega_{x} a_{x}+b_{\perp}^{2}\left(\omega_{y} a_{y}+\omega_{z} a_{z}\right)\right)
$$

Note that the cross coupling between the y-axis and z-axis disappears, since $V$ is symmetric in $\omega_{y}$ and $\omega_{z}$. Also, the last term can be rewritten as a scalar product $(\cdot)$ between a unit vector $\bar{e}=$ $\left(b_{x} \omega_{x}, b_{\perp} \omega_{y}, b_{\perp} \omega_{z}\right) / V=\left(\beta_{x} \Omega_{x}, \beta_{\perp} \Omega_{y}, \beta_{\perp} \Omega_{z}\right) / V$ and $\sum u_{i} \bar{\beta}_{i}$. Thus,

$$
H=1+\lambda \sum_{i=1}^{6} u_{i}+\bar{e} \cdot \sum_{i=1}^{6} u_{i} \bar{\beta}_{i}
$$

where

$$
\begin{array}{rlrr}
(+, 0,0): & \bar{\beta}_{1}=\left(\begin{array}{rrr}
\beta_{x}, & \beta_{\perp}, & 0
\end{array}\right) \\
(-, 0,0): & \bar{\beta}_{2}=\left(\begin{array}{rrr}
-\beta_{x}, & -\beta_{\perp}, & 0
\end{array}\right) \\
(0,+, 0): & \bar{\beta}_{3}=\left(\begin{array}{rrr}
\beta_{x}, & -\frac{1}{2} \beta_{\perp}, & \frac{\sqrt{3}}{2} \beta_{\perp}
\end{array}\right) \\
(0,-, 0): & \bar{\beta}_{4}=\left(\begin{array}{rrr}
-\beta_{x}, & \frac{1}{2} \beta_{\perp}, & -\frac{\sqrt{3}}{2} \beta_{\perp}
\end{array}\right) \\
(0,0,+): & \bar{\beta}_{5}=\left(\begin{array}{rrr}
\beta_{x}, & -\frac{1}{2} \beta_{\perp}, & -\frac{\sqrt{3}}{2} \beta_{\perp}
\end{array}\right) \\
(0,0,-): & \bar{\beta}_{6}=\left(\begin{array}{rrr}
-\beta_{x}, & \frac{1}{2} \beta_{\perp}, & \frac{\sqrt{3}}{2} \beta_{\perp}
\end{array}\right)
\end{array}
$$

We now observe that is is possible to reshape the inequality so that it will have a geometrical interpretation. Introduce

$$
h(\bar{e})=\frac{1+\lambda \sum u_{i}}{-\bar{e} \cdot \sum u_{i} \bar{\beta}_{i}}
$$

If $h \leq 1$ then $H \leq 0$ assuming that the denominator is positive. For a fixed $u$ the function $\bar{e} h(\bar{e})$ defines a flat surface perpendicular to $\sum u_{i} \bar{\beta}_{i}$. The function $h$ defines a polyhedron, where each surface corresponds to a thruster combination. The polyhedron must lie inside the unit sphere in order to satisfy the Hamiltonian inequality. Since the polyhedron is convex, it suffices to check the vertices in order to assure global satisfaction of the Hamiltonian inequality. Figure 1 shows a polyhedron corresponding to $\lambda=1$ and $\left(\beta_{x}, \beta_{\perp}\right)$ defined by $(20)$.

A thruster is switched on $\left(u_{i}=1\right)$ if

$$
\bar{e} \cdot \bar{\beta}_{i} \leq-1 / \lambda \text {. }
$$

Each thruster pair can be commanded in three possible combinations $(-, 0,+)$ since they are opposite.

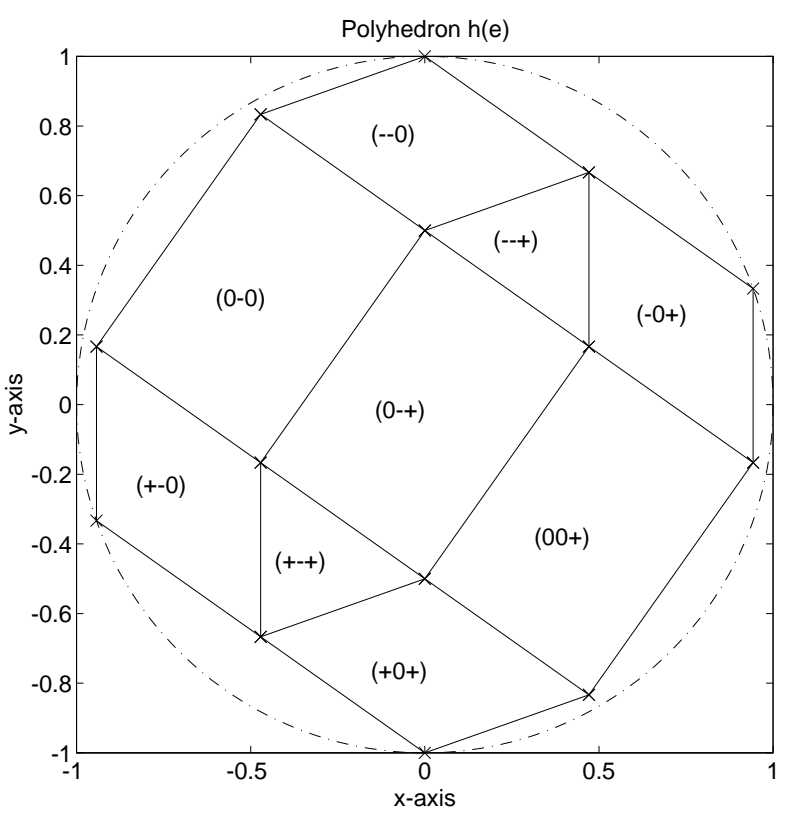

Figure 1: The polyhedron defined by $h(\bar{e})$ circumscribed by a unit sphere. Each surface corresponds to a thruster combination.

In total we have 27 combinations, of which one (all thrusters off) can be excluded, since $u_{i}=0, \forall i$ implies $H=1>0$. Thus, the polyhedron has 26 surfaces and 24 vertices.

A vertex is defined by a thruster being on $(\geq)$ and two being undefined $(=)$ in (15). The undefined thrusters could be either on or off yielding the same Lyapunov function.

We check all vertices in order to globally satisfy the Hamiltonian inequality. In total there are 24 vertices but since the configuration is symmetric, it is enough to check four since we, without loss of generality, may assume that thruster 1 is on. Out of these four conditions two are redundant and the two remaining are

Condition $1(+,+/ 0,+/ 0)$ :

$$
\begin{aligned}
e_{x} \beta_{x}+e_{y} \beta_{\perp}+1+\lambda & \leq 0 \\
e_{x} \beta_{x}-\frac{1}{2} e_{y} \beta_{\perp}+\frac{\sqrt{3}}{2} e_{z} \beta_{\perp}+\lambda & =0 \\
e_{x} \beta_{x}-\frac{1}{2} e_{y} \beta_{\perp}-\frac{\sqrt{3}}{2} e_{z} \beta_{\perp}+\lambda & =0
\end{aligned}
$$

Condition $2(+,-/ 0,-/ 0)$ :

$$
\begin{aligned}
e_{x} \beta_{x}+e_{y} \beta_{\perp}+1+\lambda & \leq 0 \\
-e_{x} \beta_{x}+\frac{1}{2} e_{y} \beta_{\perp}-\frac{\sqrt{3}}{2} e_{z} \beta_{\perp}+\lambda & =0 \\
-e_{x} \beta_{x}+\frac{1}{2} e_{y} \beta_{\perp}+\frac{\sqrt{3}}{2} e_{z} \beta_{\perp}+\lambda & =0
\end{aligned}
$$


These two conditions together with $e_{x}^{2}+e_{y}^{2}+e_{z}^{2}=1$ can be synthesized into the following inequalities

$$
\begin{gathered}
\frac{(1+3 \lambda)^{2}}{\beta_{x}^{2}}+\frac{4}{\beta_{\perp}^{2}} \leq 9 \\
\frac{(1-\lambda)^{2}}{\beta_{x}^{2}}+\frac{4(1+2 \lambda)^{2}}{\beta_{\perp}^{2}} \leq 9
\end{gathered}
$$

The two conditions specify a set of $\left(\beta_{x}, \beta_{\perp}\right)$. Every member in this set defines a Lyapunov function, $V_{\beta}$, that satisfies $H \leq 0$. By combining these Lyapunov functions within this set a new global Lyapunov function, $V_{*}$, can be obtained by minimizing $V_{\beta}$ with respect to $\beta$.

\subsection{Minimizing $V_{\beta}$}

We proceed as follows: for each $\left(\Omega_{x}, \Omega_{\perp}\right)$ there exists an optimal $\left(\beta_{x}, \beta_{\perp}\right)$, possibly not unique. Thus,

$$
V_{*}\left(\Omega_{x}, \Omega_{\perp}\right)=\min _{\beta_{x}, \beta_{\perp}} V_{\beta}\left(\Omega_{x}, \Omega_{\perp}\right)
$$

with

$$
V_{\beta}\left(\Omega_{x}, \Omega_{\perp}\right)=\sqrt{\left(\beta_{x} \Omega_{x}\right)^{2}+\left(\beta_{\perp} \Omega_{\perp}\right)^{2}} .
$$

The two conditions (16) and (17) coincide in

$$
\begin{aligned}
& \beta_{x}^{2}=\lambda^{2}+\frac{2}{3} \lambda+\frac{1}{3} \\
& \beta_{\perp}^{2}=2 \lambda^{2}+\frac{4}{3} \lambda+\frac{2}{3} .
\end{aligned}
$$

Note that $\beta_{\perp}=\sqrt{2} \beta_{x}$.

We get three solutions to the minimization problem: one corresponding to the intersection defined by (20), and two on each side of the intersection where either condition is limiting.

When condition (16) is limiting (equality) we obtain

$$
\begin{array}{r}
V^{2}(\bar{\Omega})=\beta_{x}^{2} \Omega_{x}^{2}+\beta_{\perp}^{2} \Omega_{\perp}^{2} \\
=\left(\beta_{x}^{2} \Omega_{x}^{2}+\beta_{\perp}^{2} \Omega_{\perp}^{2}\right)\left(\frac{(1+3 \lambda)^{2}}{9 \beta_{x}^{2}}+\frac{4}{9 \beta_{\perp}^{2}}\right) \\
=\left(\frac{1+3 \lambda}{3}\left|\Omega_{x}\right|+\frac{2}{3} \Omega_{\perp}\right)^{2} \\
+\left(\frac{2 \beta_{x}}{3 \beta_{\perp}}\left|\Omega_{x}\right|-\frac{(1+3 \lambda) \beta_{\perp}}{3 \beta_{x}} \Omega_{\perp}\right)^{2} \\
\leq\left(\frac{1+3 \lambda}{3}\left|\Omega_{x}\right|+\frac{2}{3} \Omega_{\perp}\right)^{2}
\end{array}
$$

with equality for

$$
\left(\frac{\beta_{x}}{\beta_{\perp}}\right)^{2}=\frac{1+3 \lambda}{2} \frac{\Omega_{\perp}}{\left|\Omega_{x}\right|} .
$$

Thus

$$
V_{1}=\frac{1+3 \lambda}{3}\left|\Omega_{x}\right|+\frac{2}{3} \Omega_{\perp} .
$$

Similarly, when condition (17) is limiting we obtain

$$
V_{2}=\frac{|1-\lambda|}{3}\left|\Omega_{x}\right|+\frac{2+4 \lambda}{3} \Omega_{\perp}
$$

for

$$
\left(\frac{\beta_{x}}{\beta_{\perp}}\right)^{2}=\frac{|1-\lambda|}{2+4 \lambda} \frac{\Omega_{\perp}}{\left|\Omega_{x}\right|}
$$

The intersection (20) yields

$$
V_{3}=\sqrt{\left(\lambda^{2}+\frac{2}{3} \lambda+\frac{1}{3}\right)\left(\Omega_{x}^{2}+2 \Omega_{\perp}^{2}\right)} .
$$

Depending on $\Omega_{x}$ and $\Omega_{\perp}$ we select one of the three Lyapunov functions.

The synthesized Lyapunov function of the problem becomes

$$
V(\bar{\Omega})= \begin{cases}V_{1}(\bar{\Omega}) & (1+3 \lambda) \Omega_{\perp} \leq\left|\Omega_{x}\right| \\ V_{2}(\bar{\Omega}) & |1-\lambda| \Omega_{\perp} \geq(1+2 \lambda)\left|\Omega_{x}\right| \\ V_{3}(\bar{\Omega}) & \text { otherwise. }\end{cases}
$$

Note that if $\lambda=0$ only $V_{1}$ and $V_{2}$ can apply; if $\lambda=1$ only $V_{1}$ and $V_{3}$.

\subsection{Recovering the Controller}

The control law can be derived using (26) and by minimizing $H$ with respect to $u$. Alternatively, $\beta_{x}$ and $\beta_{\perp}$ can be solved for and (15) can be used.

In order to get a practicable control law we also need some sort of stopping criterion or fine-pointing mode; this will not be discussed here.

\subsection{Finding Lower Bounds}

We can also provide a lower bound for the example given in the previous section. Using the same reasoning as for the upper bound we want to find a loss function $V$ that assures $H \geq 0$, or equivalently $h \geq 1$. One such lower bound can be found by finding the largest sphere inscribed in the polyhedron defined by $h(\bar{e})$. Assume for simplicity that $\lambda=1$. In that case it can be shown that $J(\bar{\Omega}) \geq \sqrt{\frac{4}{3} \Omega_{x}^{2}+\frac{8}{3} \Omega_{\perp}^{2}}$.

This bound can be further elaborated by parameter optimization using the same technique as for the upper bound. Thus, we have provided both upper and lower bounds for the cost criterion, $J$. If the candidate loss function is parametrized, it can be optimized in each point locally for obtaining an improved global bound. 


\section{Conclusions}

In this paper we have applied a Lyapunov technique for deriving a feedback control law with guaranteed performance bounds. The technique is based on the Hamilton-Jacobi-Bellman equation. By assuming a parametrized set of Lyapunov functions satisfying a Hamiltonian inequality a control law is derived. Usually it is difficult to assure global satisfaction of the Hamiltonian inequality but with a proper choice of the Lyapunov function this may be alleviated.

\section{References}

[1] G. Leitmann. The Calculus of Variation and Optimal Control. Plenum Press, 1981.

[2] D. Hill and P. Moylan. The stability of nonlinear dissipative systems. IEEE Transactions on Automatic Control, AC-21:708-711, 1976.

[3] D. Hill and P. Moylan. Connection between finite gain and assymptotic stability. IEEE Transactions on Automatic Control, AC-25:931-936, 1980.

[4] J. C. Willems. The generation of Lyapunov functions for input-output stable systems. SIAM Journal of Control, 9:105-133, 1971. 